\title{
Lysine Inhibition of in vivo Homocitrate Synthesis in Penicillium chrysogenum*
}

\author{
By A. L. DEMAIN AND P. S. MASUREKAR† \\ Department of Nutrition and Food Science, Massachusetts Institute of Technology, \\ Cambridge, Massachusetts 02139 , U.S.A.
}

(Received 28 September 1973)

\section{SUMMAR Y}

A lysine bradytroph of Penicillium chrysogenum Wis. 54-I 255 was isolated. The mutant $\left(\mathrm{L}_{1}\right)$ grew with $\alpha$-aminoadipic acid and excreted homocitrate. In vivo accumulation of homocitrate, which did not require protein synthesis, was markedly reduced by lysine. These results indicate that lysine diminution of penicillin production is caused by feedback inhibition of homocitrate synthase, thus limiting the supply of $\alpha$-aminoadipic acid available for penicillin biosynthesis.

\section{INTRODUCTION}

For many years it has been known that lysine decreases the degree of penicillin biosynthesis in Penicillium chrysogenum (Demain, 1957). Since a biosynthetic lysine precursor, $\mathrm{L}-\alpha$-aminoadipic acid, is involved in penicillin formation (Arnstein \& Morris, 1960), and since $\alpha$-aminoadipic acid can reverse lysine diminution of penicillin formation (Somerson, Demain \& Nunheimer, I96I), we postulated that a branched biosynthetic pathway was responsible for production of lysine and penicillin and that the 'lysine effect' was due to negative feedback control of an enzyme of the early common pathway (Demain, 1966). Goulden \& Chattaway (1968) provided supporting evidence by showing that the concentration of $\alpha$-aminoadipic acid in the intracellular pool of a late-blocked mutant of $P$. chrysogenum is low until lysine is depleted. Masurekar \& Demain (1972) showed that, although prior growth in lysine stimulated penicillin formation by washed suspensions of prototrophic $P$. chrysogenum, the presence of lysine in the resting-cell suspension markedly inhibited penicillin biosynthesis. They concluded that the 'lysine effect' was caused by feedback inhibition of an early enzyme of lysine biosynthesis.

To determine whether homocitrate synthase, the initial lysine biosynthetic enzyme, is inhibited by lysine, we needed an early-blocked lysine auxotroph. Lysine auxotrophs were isolated (Masurekar, Kahgan \& Demain, 1972), one of which was an early lysine bradytroph. We show here that in vivo homocitrate accumulation by this leaky auxotroph is markedly inhibited by lysine.

\section{METHODS}

Cultures. Nine lysine auxotrophs were isolated from Penicillium chrysogenum Wis. 54I 255 after $2 \mathrm{~h}$ ethylmethane sulphonate mutagenesis followed by pentachlorophenate enrichment (Masurekar et al. 1972). The lysine requirement of only one mutant, a leaky auxotroph, could be satisfied by $\alpha$-aminoadipic acid. This bradytroph, designated as $L_{1}$,

* Contribution No. 2250 from the Department of Nutrition and Food Science, Massachusetts Institute of Technology, Cambridge, Massachusetts 02139, U.S.A.

$\dagger$ Present address: Research Laboratories, Eastman Kodak Co., Rochester, New York 14650, U.S.A. 
thus appears to be partially blocked before $\alpha$-aminoadipic acid. The remaining auxotrophs presumably are either blocked between $\alpha$-aminoadipic acid and lysine or unable to take up $\alpha$-aminoadipic acid.

Penicillium chrysogenum strains Wis. 54-I255 and $\mathrm{L}_{1}$ were maintained on slants of Sabouraud dextrose agar (Difco) with $0.1 \%$ yeast extract.

Identification of accumulation product of mutant $\mathrm{L}_{1}$. Mutant $\mathrm{L}_{1}$ and its parent (Wis. 54-I 255) were grown for 3 days on the shaker at $25^{\circ} \mathrm{C}$ in the glucose-citrate medium (citrate No. 2 medium of Yamamoto \& Segel, I966) supplemented with $250 \mu \mathrm{g}$ of L- $\alpha$-aminoadipic acid/ $\mathrm{ml}$. The mycelia were filtered (Schleicher and Schuell No. 595 paper) and washed three times with a total of $6 \mathrm{vol}$. of sterile distilled water. The washed mycelia were resuspended in low glucose-citrate medium, i.e. glucose-citrate medium with glucose reduced from $40 \mathrm{~g}$ to $\mathrm{I0} \mathrm{g} / \mathrm{l}$; this reduction in the amount of sugar facilitated the subsequent concentration and extraction of the filtrates. To the medium, $0.5 \mu \mathrm{Ci}$ sodium $\left[\mathrm{I}^{14} \mathrm{C}\right]$ acetate $/ \mathrm{ml}$ was added. After $24 \mathrm{~h}$ of incubation on the shaker, the mycelia were removed by filtration and the clear filtrates were acidified with $\mathrm{HCl}$ to $\mathrm{pH} 2 \cdot 0$. The filtrates were concentrated in vacuo in a rotary evaporator to $5 \mathrm{ml}$, and $\mathrm{I} \mathrm{ml}$ of the concentrated solutions was extracted with $3 \mathrm{ml}$ of ether by vigorously mixing on a vortex mixer. The organic and aqueous phases were separated in a small separatory funnel.

The ether extracts were chromatographed on paper in the following solvent systems (Bhattacharjee \& Strassman, 1967a): (I) ethyl ether-benzene-formic acid-water (21:9:7:2); (2) I-butanol-acetic acid-water (4:I:I); (3) 3-butanol-methyl ethyl ketone-formic acidwater (40:30:I5:I5); (4) I-butanol-formic acid-water (5:I:I). After development, the strips were dried and examined in a Packard radio-chromatogram scanner to locate the radioactive areas. These strips were also sprayed with neutralized $0.04 \%$ bromphenol blue in $95 \%$ ethanol. This indicator reveals acids as yellow spots on a blue background. By the combination of scanning and spraying, radioactive acids were detected. Homocitric lactone, citric acid, $\alpha$-ketoglutaric acid and succinic acid were spotted as standards. For co-chromatography, the ether extract of the broth of mutant $L_{1}$ was chromatographed in solvent system I and peaks corresponding to the homocitric acid and its lactone were located by scanning. In this solvent system, the faster-moving component is the lactone and the slower-moving component is the free acid. The portion of the strip corresponding to homocitric lactone was cut out and eluted with $95 \%$ ethanol into a test tube, the elution being carried out in a desiccator saturated with ethanol. The eluate was concentrated in vacuo in a rotary evaporator and was co-chromatographed with authentic homocitric lactone in solvent systems 2, 3 and 4 . The chromatograms were scanned and were sprayed with bromphenol blue for visualization of acid spots.

In vivo accumulation of homocitrate. Mutant $\mathrm{L}_{1}$ conidia from a slant were inoculated into $50 \mathrm{ml}$ of Sabouraud dextrose broth (Difco) supplemented with $0 . \mathrm{I} \%$ yeast extract in $250 \mathrm{ml}$ Erlenmeyer flasks. After $48-72 \mathrm{~h}$ of growth, the mycelia were filtered and washed. The washed mycelia were resuspended in $50 \mathrm{ml}$ of sterile distilled water and served as inoculum. Fifty $\mathrm{ml}$ of glucose-citrate medium supplemented with $400 \mu \mathrm{g}$ lysine. $\mathrm{HCl} / \mathrm{ml}$ in $250 \mathrm{ml}$ Erlenmeyer flasks were inoculated with $2 \mathrm{ml}$ of mutant $\mathrm{L}_{1}$ inoculum. After $72 \mathrm{~h}$ of growth, the mycelia were harvested, washed and resuspended in low glucose-citrate medium. Sodium $\left[{ }^{-14} \mathrm{C}\right]$ acetate $(0.5 \mu \mathrm{Ci} / \mathrm{ml})$ was added and the flasks were shaken for $24 \mathrm{~h}$. The mycelia were harvested by filtration and dry mycelial weight was determined. Homocitrate accumulation in the filtrate was measured by paper chromatography in solvent system $\mathrm{I}$. The chromatograms were examined on the radioactive chromatogram scanner to locate the two radioactive peaks corresponding to homocitric acid and its lactone. The portions of the 
chromatogram corresponding to these peaks were cut out and counted in a Beckman scintillation spectrometer, with toluene scintillation fluid [4 $\mathrm{g}$ of Omnifluor (New England Nuclear, Boston, Massachusetts, U.S.A.) in I 1 reagent-grade toluene].

We have used the incorporation of labelled acetate into homocitrate to indicate the in vivo activity of homocitrate synthase. It is, however, realized that we are only dealing with an approximation, since the amount of homocitrate formed is not necessarily directly proportional to the incorporation of $\left[\mathrm{I}^{-14} \mathrm{C}\right]$ acetate into homocitrate. Direct proportionality exists only when the specific radioactivity of the intracellular acetate involved in synthesis (i.e. endogenous plus that from the extracellular fluid) is constant throughout the experiment.

Inhibition of protein synthesis by cycloheximide. The mycelium of mutant $\mathbf{L}_{\mathbf{1}}$, grown in glucose-citrate-lysine medium, was suspended in the low glucose-citrate medium containing 100000 c.p.m. L-[U-14 C]proline/ml (specific activity $195 \mathrm{mCi} / \mathrm{mmol}$ ) with and without $\mathrm{IO}^{-4} \mathrm{M}-$ cycloheximide. Samples were withdrawn periodically and the mycelia were treated with $20 \mathrm{ml}$ of $5 \%$ cold trichloroacetic acid for $30 \mathrm{~min}$. The mycelia were collected by filtration through Schleicher and Schuell 895-E filter paper and washed 3 times with a total of 3 vol. of $5 \%$ cold trichloroacetic acid containing $50 \mu \mathrm{g}$ unlabelled L-proline $/ \mathrm{ml}$. To the mycelia were added I $\mathrm{ml}$ of water and $15 \mathrm{ml}$ of Bray's solution (Bray, 1960). Radioactivity was determined in a Beckman scintillation spectrometer. After being counted, the mycelia were collected on tared Schleicher and Schuell 895-E filter papers and washed, first with acetone to remove the scintillation fluid, and then with water. The filter paper discs were dried under an infrared lamp and weighed to determine dry mycelial weight.

Penicillin fermentation. Inoculum of Penicillium chrysogenum $\mathrm{L}_{1}$ was prepared from conidia as described above. Two $\mathrm{ml}$ of inoculum were added to defined seed medium ( $50 \mathrm{ml}$ in a 250 Erlenmeyer flask) containing (per litre): $40 \mathrm{~g}$ glucose, $13 \mathrm{~g}\left(\mathrm{NH}_{4}\right)_{2} \mathrm{SO}_{4}, 0.4 \mathrm{~g} \mathrm{~L}$ lysine. $\mathrm{HCl}, \mathrm{I} 3 \mathrm{~g} \mathrm{CaCO}_{3}$ (sterilized separately), and $100 \mathrm{ml}$ salts mixture. The salts mixture contained (per litre): $30 \mathrm{~g} \mathrm{KH}_{2} \mathrm{PO}_{4}, 5 \mathrm{~g} \mathrm{Na}_{2} \mathrm{SO}_{4}, 2.5 \mathrm{~g} \mathrm{MgSO}_{4} \cdot 7 \mathrm{H}_{2} \mathrm{O}$, I.0 g FeSO ${ }_{4} \cdot 7 \mathrm{H}_{2} \mathrm{O}$, $0.5 \mathrm{~g} \mathrm{CaCl}_{2} .2 \mathrm{H}_{2} \mathrm{O}, 0.2 \mathrm{~g} \mathrm{MnSO}_{4} . \mathrm{H}_{2} \mathrm{O}, 0.2 \mathrm{~g} \mathrm{ZnSO}_{4} .7 \mathrm{H}_{2} \mathrm{O}$ and $0.05 \mathrm{~g} \mathrm{CuSO}_{4} .5 \mathrm{H}_{2} \mathrm{O}$. After $72 \mathrm{~h}$ on the shaker, $10 \mathrm{ml}$ of seed were added to $250 \mathrm{ml}$ flasks containing $40 \mathrm{ml}$ of defined penicillin-production medium. The production medium was composed of (per litre): $30 \mathrm{~g}$ lactose, $10 \mathrm{~g}$ glucose, $6 \mathrm{~g}$ ammonium lactate, $3.5 \mathrm{~g}$ ammonium acetate, $\mathrm{I} \cdot 8 \mathrm{~g}$ phenylacetylethanolamine, $0.4 \mathrm{~g} \mathrm{~L}$-lysine. $\mathrm{HCl}$, $\mathrm{m} \mathrm{ml}$ oleic acid and $100 \mathrm{ml}$ salts mixture. The sugars were sterilized separately. At various times during the fermentation, flasks were removed and the mycelia harvested by filtration, washed and placed in a suspension medium containing 0.5 $\mu \mathrm{Ci}$ of sodium $\left[\mathrm{I}^{-14} \mathrm{C}\right]$ acetate $/ \mathrm{ml}$ with and without $20 \mathrm{~mm}$-lysine. The suspension medium contained (per litre): $48 \mathrm{~g}$ lactose (sterilized separately), $\mathrm{r} \cdot 6 \mathrm{~g}$ sodium phenylacetate, $320 \mathrm{ml}$ I M-sodium phosphate buffer ( $\mathrm{pH} 7)$ and $\mathrm{I} 60 \mathrm{ml}$ salts mixture. After $24 \mathrm{~h}$ of incubation, the mycelia were harvested for the determination of dry mycelial weight and homocitrate accumulated in the broth was measured. Penicillin was measured by the hydroxamate method (Boxer \& Everett, 1949) at the time of harvest of mycelium from the production medium.

Dry mycelial weight determination. The harvested mycelia were homogenized in a highspeed blender (Omnimixer, Sorvall and Co.) at $17000 \mathrm{rev} . / \mathrm{min}$ for $2 \mathrm{~min}$. The absorbance of the homogenized mycelial suspension was measured in a Klett-Summerson photoelectric colorimeter with red filter (No. 66) after proper dilution. Dry mycelial weight was calculated from a calibration curve of absorbance against dry weight.

Chemicals. Cycloheximide, $\alpha$-ketoglutaric acid and $\alpha$-aminoadipic acid were purchased from Calbiochem. Sodium $\left[\mathrm{I}^{14} \mathrm{C}\right]$ acetate, specific activity $5 \mathrm{I}-57 \mathrm{mCi} / \mathrm{mmol}$, was obtained from New England Nuclear. Phenylacetylethanolamine and benzylpenicillin were generously 
Table I. $R_{k}$ values of the product of the mutant and authentic homocitric lactone, citric acid, $\alpha$-ketoglutaric acid and succinic acid

$\begin{array}{cccccc}\begin{array}{c}\text { Solvent } \\ \text { system } \\ \text { no. }\end{array} & \begin{array}{c}\text { Mutant } \\ \text { product }\end{array} & \begin{array}{c}\text { Homocitric } \\ \text { lactone }\end{array} & \begin{array}{c}\text { Citric } \\ \text { acid }\end{array} & \begin{array}{c}\alpha \text {-Keto- } \\ \text { glutaric } \\ \text { acid }\end{array} & \begin{array}{c}\text { Succinic } \\ \text { acid }\end{array} \\ \text { I } & 0.25,0.45 & 0.42 & 0.18 & 0.83 & 0.96 \\ 2 & 0.63 & 0.61 & 0.54 & - & 0.94 \\ 3 & 0.59,0.72 & 0.70 & 0.53 & 0.84 & 0.87 \\ 4 & 0.54,0.63 & 0.63 & 0.48 & 0.87 & 0.87\end{array}$

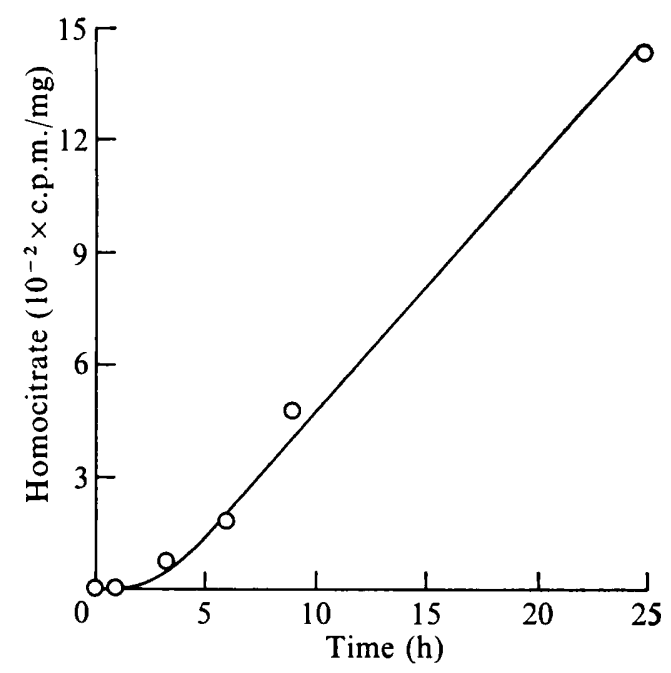

Fig. I. Time-course of in vivo formation of homocitrate from sodium $\left[{ }^{1-14} \mathrm{C}\right]$ acetate by Penicillium chrysogenum $\mathbf{L}_{\mathbf{1}}$.

donated by Dr D. W. Gallup and Dr J. Birnbaum of Merck and Co., respectively. Homocitric lactone was kindly supplied by $\mathrm{Dr} \mathrm{H}$. Broquist (Vanderbilt University, Nashville, Tennessee, U.S.A.) and by Dr A. Tucci (Albert Einstein Medical Centre, Philadelphia, Pennsylvania, U.S.A.).

\section{RESULTS}

Accumulation product of mutant $\mathrm{L}_{1}$. The ether extracts from mutant $\mathrm{L}_{1}$ and the prototrophic parent Wis $54-1255$ were chromatographed in four solvent systems as described under Methods. The chromatograms were scanned to detect radioactive peaks and were sprayed with bromphenol blue to visualize the acid spots. When the chromatograms developed in solvent systems I, 3 and 4 were examined, the chromatograms for mutant $\mathrm{L}_{1}$ were found to contain two radioactive acid spots not present in those of the parent. The $R_{F}$ values of the faster-migrating spot were similar to those obtained for authentic homocitric lactone in the same solvent systems (Table I). In solvent systems I, 3 and 4 , homocitric acid and its lactone migrate at different rates; the faster-moving component is the lactone and the slower-moving component is the free acid. In solvent system 2, both migrate as a single spot and the $R_{F}$ is the same as that of the single extra radioactive acid spot of mutant $\mathrm{L}_{1}$ (Table $\mathrm{I}$ ). To confirm these observations, we eluted the mutant peak 


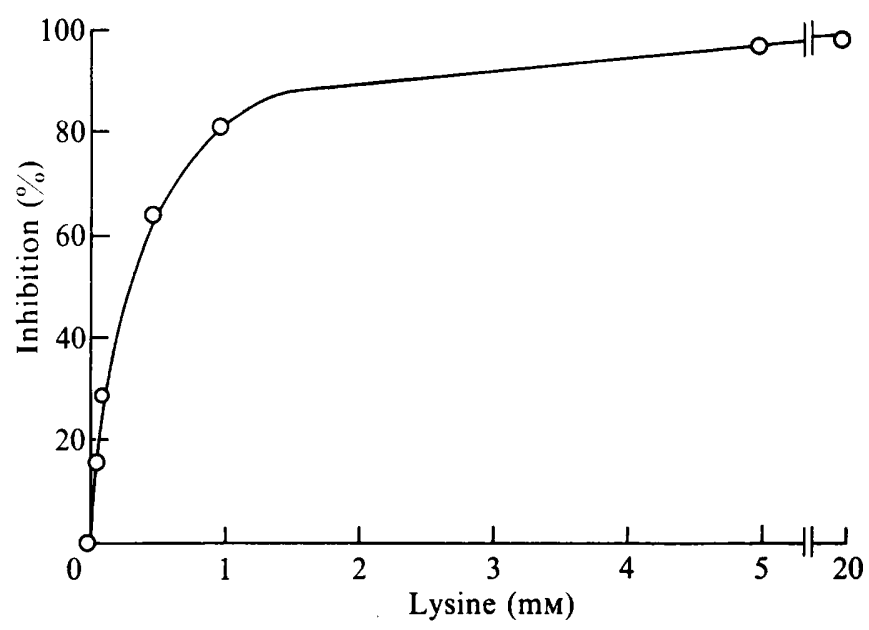

Fig. 2. Inhibition of in vivo biosynthesis of homocitrate by lysine in Penicillium chrysogenum $\mathbf{L}_{\mathbf{1}}$.

Table 2. Effect of cycloheximide on the in vivo synthesis of homocitrate

Cycloheximide
concentration

(M)

0
Homocitrate

(c.p.m./mg dry mycelial wt)

235

201

corresponding to homocitric lactone with ethanol from the chromatograms developed in solvent system I. The ethanol extract was co-chromatographed with authentic unlabelled homocitric lactone in solvent systems 2, 3 and 4 . The $R_{F}$ values of the radioactive peaks and the acid spots were identical. These results confirm that the mutant accumulates homocitrate.

In vivo synthesis of homocitrate. Studies on the time-course of in vivo homocitrate synthesis were carried out by growing mutant $\mathrm{L}_{\mathbf{1}}$ in the glucose-citrate-lysine medium and resuspending the mycelium in the low glucose-citrate medium containing sodium $\left[\mathrm{I}-{ }^{14} \mathrm{C}\right]$ acetate. The flasks were removed periodically and the label incorporated into homocitrate was measured. The results (Fig. I) showed a linear formation of homocitrate from 3 to $24 \mathrm{~h}$. For the remaining experiments, in vivo synthesis of homocitrate was determined after $24 \mathrm{~h}$.

The relatively slow in vivo formation of homocitrate made it important to determine whether protein synthesis was involved or whether homocitrate was produced by preformed enzymes. For this purpose, we measured homocitrate production in the presence and absence of $\mathrm{IO}^{-4} \mathrm{M}$-cycloheximide. This concentration of cycloheximide was sufficient to inhibit protein synthesis as determined by inhibition of the incorporation of $\left[{ }^{14} \mathrm{C}\right]$ proline into the trichloroacetic acid-insoluble fraction. Table 2 shows that there is no difference in the amount of homocitrate produced in the presence or in the absence of cycloheximide. Hence protein synthesis is not required for the accumulation of homocitrate in the $24 \mathrm{~h}$ suspension system, which can thus be used to investigate lysine inhibition of homocitrate synthesis.

Effect of lysine on in vivo homocitrate synthesis. The effect of lysine was examined by determining homocitrate formation in the low glucose-citrate medium supplemented with 


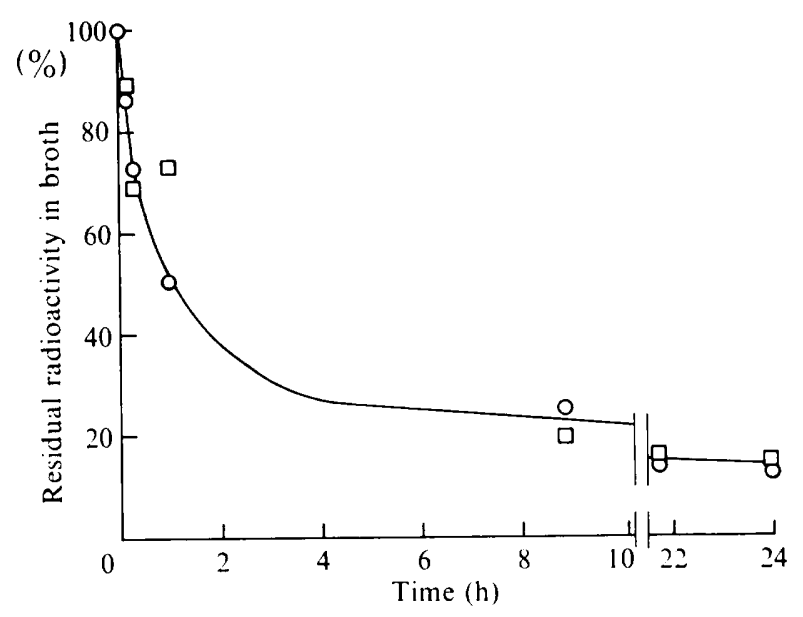

Fig. 3. Lack of lysine effect on uptake of sodium $\left[{ }_{1}-{ }^{14} \mathrm{C}\right]$ acetate by Penicillium chrysogenum $\mathbf{L}_{\mathbf{1}}$. $O$, With 20 mM-lysine; $\square$, without lysine.

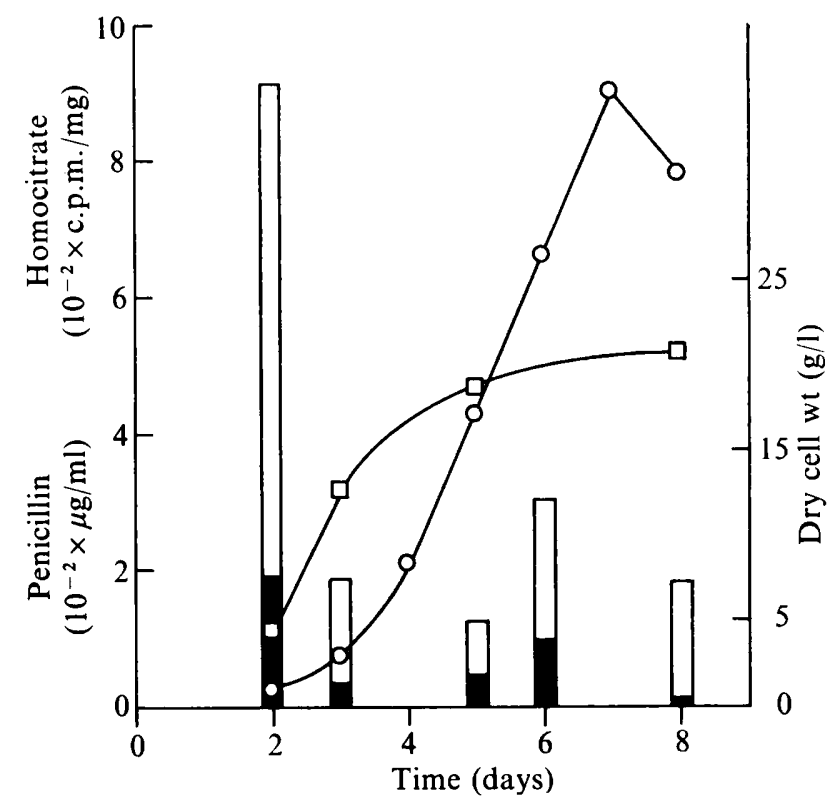

Fig. 4. Ability of Penicillium chyrsogenum $\mathrm{L}_{1}$ mycelia taken at various times from a penicillin fermentation to produce homocitrate from sodium $\left[\mathrm{I}^{14} \mathrm{C}\right]$ acetate. Penicillin was measured at the time of harvest of the mycelium from defined production medium. Dry mycelial weight was determined after $24 \mathrm{~h}$ incubation in the suspension medium. Homocitrate was measured at the end of the $24 \mathrm{~h}$ incubation in the suspension medium. It is expressed on the basis of dry cell weight. Open bar, homocitrate synthesis in the absence of lysine; closed bar, homocitrate synthesis in the presence of lysine. $\square-\square$, Dry cell weight; $\bigcirc-O$, penicillin. 
various concentrations of lysine. The results shown in Fig. 2 demonstrate that lysine strongly inhibited the in vivo formation of homocitrate. In this experiment, $50 \%$ inhibition occurred at a lysine concentration of $0.3 \mathrm{~mm}$.

To make sure that lysine did not interfere with the transport of labelled acetate into the mycelium, we measured the uptake of radioactivity from the medium during suspension in the low glucose-citrate medium. As shown in Fig. 3, there was no difference in the uptake of acetate in the presence or in the absence of $20 \mathrm{~mm}$-lysine. Thus, lysine reduced accumulation of homocitrate by an inhibitory action inside the cell, presumably by inhibiting homocitrate synthase.

In vivo homocitrate-synthesizing activity at various phases of a penicillin fermentation. Since the results described above showed that lysine inhibits in vivo homocitrate formation, we felt it desirable to determine the homocitrate-synthesizing activity and its sensitivity to inhibition by lysine at various times in a medium capable of supporting penicillin production. Penicillin, a secondary metabolite, is produced in a distinct phase of the fermentation (idiophase) subsequent to the growth phase (trophophase). Mutant $L_{1}$ was useful for such an experiment, since its 'leakiness' allows it to produce penicillin as well as accumulate homocitrate.

Mutant $L_{1}$ was grown in the defined penicillin production medium containing lysine. At various times, flasks were removed, the mycelia harvested and divided between flasks of a lactose-phenylacetate suspension medium either containing or lacking lysine. Penicillin was measured at the time of the harvest of the mycelia from the production medium. Homocitrate and dry mycelial weight were determined at the end of a $24 \mathrm{~h}$ suspension period. The results of this experiment are shown in Fig. 4. Homocitrate-synthesizing activity was maximal during the trophophase and decreased drastically during the idiophase. The activity in both phases was strongly ( 65 to $95 \%$ ) inhibited by lysine.

\section{DISCUSSION}

The distribution of lysine auxotrophs isolated after ethylmethane sulphonate mutagenesis was somewhat surprising in that eight out of the nine auxotrophs appeared to be blocked late in lysine biosynthesis, i.e. after aminoadipic acid. Fortunately, the ninth mutant $\left(\mathrm{L}_{1}\right)$ was blocked early in the sequence and accumulated homocitrate.

Previous studies with other fungi on the in vivo accumulation of intermediates of the lysine biosynthetic pathway and the effect of lysine on such accumulation have contributed significantly to our understanding of the regulation of lysine biosynthesis (Bhattacharjee \& Strassman, $1967 a, b)$. Control of lysine biosynthesis via feedback regulation occurs in Saccharomyces, Rhodotorula and Neurospora (Trupin \& Broquist, 1965; Maragoudakis, Holmes \& Strassman, 1967; Bhattacharjee, 1970; Glass \& Bhattacharjee, 197I). Lysine produces feedback repression of homocitrate synthase (Hogg \& Broquist, 1968; Tucci, I969) and aminoadipic semialdehyde-glutamate reductase (Sinha, Kurtz \& Bhattacharjee, 197I) and feedback inhibition of homocitrate synthase (Maragoudakis et al. 1967; Hogg $\&$ Broquist, I968). The present studies showed that lysine inhibits homocitrate synthase activity in Penicillium chrysogenum.

Penicillium chrysogenum mutant $\mathrm{L}_{1}$, an early-blocked lysine bradytroph, accumulates homocitrate rather slowly and we chose a duration of $24 \mathrm{~h}$ for our experiments. Because of this long duration, we felt that diminution of homocitrate formation by lysine could be explained by repression of a homocitrate synthase undergoing rapid turnover, or by feedback inhibition. We found, however, that cycloheximide did not diminish homocitrate formation, 
thus proving that the normal production of homocitrate in our $24 \mathrm{~h}$ system did not involve protein synthesis. In view of this finding, diminution of homocitrate formation by lysine cannot be due to lysine repression of homocitrate synthase; feedback inhibition is apparently the true mechanism.

Homocitrate-synthesizing activity in Penicillium chrysogenum was maximal during the rapid growth phase and drastically decreased as the growth slowed down. A number of enzymes of primary metabolism showed similar behaviour (Kisumi, Komatsubara \& Chibata, I97I; Hostalek et al. 1969; Hampton, McCormick, Behforouz \& Freese, 197I). There are two aspartokinases in Bacillus subtilis (Hampton et al. 197I), only one of which is sensitive to lysine inhibition. As growth slows down, the level of the sensitive enzyme decreases while that of insensitive aspartokinase (which is low during rapid growth) increases. In the case of homocitrate synthase in Penicillium chrysogenum, such a dramatic difference in regulation is not readily apparent since lysine strongly inhibits in vivo homocitrate accumulation in cells taken from both the growth and the penicillin-production phases. There was some variation in the degree of inhibition observed at the various times; this could be due to fluctuations in the level of the intracellular lysine pool or to experimental variation. An important implication of these observations is that penicillin synthesis should be extremely sensitive to lysine present during the production phase.

This work was supported by Public Health Service Grant AI-09345 from the National Institute of Allergy and Infectious Diseases. We thank D. I. C. Wang and A. J. Sinskey for their interest in this work. We are pleased to acknowledge the helpful suggestions of G. M. Brown and the use of his laboratory facilities for certain experiments.

\section{REFERENCES}

ARNSTEIN, H. R. V. \& MoRris, D. (1960). The structure of a peptide containing $\alpha$-aminoadipic acid, cystine and valine, present in the mycelium of Penicillium chrysogenum. Biochemical Journal 76, 357-36I.

BHATTACHARJEE, J. K. (1970). Leaky mutation and coordinate regulation of the accumulation of lysine precursors in Saccharomyces. Canadian Journal of Genetics and Cytology 12, 785-789.

BhattacharJeE, J. K. \& Strassman, M. (1967 $a$ ). Accumulation of tricarboxylic acids related to lysine biosynthesis in a yeast mutant. Journal of Biological Chemistry 242, 2542-2546.

BhattacharjeE, J. K. \& Strassman, M. (1967 $b$ ). Accumulation of $\alpha$-ketoadipic acid by a lysine-requiring yeast mutant. Journal of the Albert Einstein Medical Center 15, 44-49.

BoXER, G. M. \& EVERETT, P. M. (1949). Colorimetric determination of benzylpencillin. Colorimetric determination of total penicillins. Analytical Chemistry 2r, 670-673.

BRAY, G. (1960). A simple efficient liquid scintillator for counting aqueous solutions in a liquid scintillation counter. Analytical Biochemistry r, 279-285.

Demain, A. L. (1957). Inhibition of penicillin formation by lysine. Archives of Biochemistry and Biophysics 67, 244-245.

Demain, A. L. (1966). Biosynthesis of penicillins and cephalosporins. In Biosynthesis of Antibotics, vol. I, pp. 29-64. Edited by J. Snell. New York: Academic Press.

Glass, J. \& BhattacharJee, J. K. (1971). Lysine biosynthesis in Rhodotorula: accumulation of homocitric, homoaconitic and homoisocitric acids in a leaky mutant. Genetics 67, 365-376.

Goulden, S. A. \& Chattaway, F. W. (1968). Lysine control of $\alpha$-aminoadipate and penicillin synthesis in Penicillium chrysogenum. Biochemical Journal I10, 55 P.

Hampton, M. L., McCormick, N. G., Behforouz, N. C. \& Freese, E. (197I). Regulation of two aspartokinases in Bacillus subtilis. Journal of Bacteriology ro8, I $129-1134$.

HogG, R. W. \& Broquist, H. P. (1968). Homocitrate formation in Neurospora crassa. Relation to lysine biosynthesis. Journal of Biological Chemistry 243, 1839-1845.

Hostalek, Z., Tinterova, M. Jechova, V., Blumauerova, M., Suchy, J. \& Vanek, Z. (i969). Regulation of biosynthesis of secondary metabolites. I. Biosynthesis of chlortetracycline and tricarboxylic acid cycle activity. Biotechnology and Bioengineering II, 539-548. 
Kisumi, M., Komatsubara, S. \& Chibata, I. (1971). Valine accumulation by $\alpha$-aminobutyric acid resistant mutants of Serratia marcescens. Journal of Bacteriology 106, 493-499.

Maragoudakis, M. E., Holmes, H. \& Strassman, M. (1967). Control of lysine biosynthesis in yeast by a feedback mechanism. Journal of Bacteriology 93, 1677-1680.

Masurekar, P. S. \& Demain, A. L. (1972). Lysine control of penicillin biosynthesis. Canadian Journal of Microbiology $\mathbf{1 8}$, 1045-1048.

Masurekar, P. S., Kahgan, M. P. \& Demain, A. L. (1972). Mutagenesis and enrichment of autotrophs in Penicillium chrysogenum. Applied Microbiology 24, 995-996.

Sinha, A. K., Kurtz, M. \& Bhattacharjee, J. K. (1971). Effect of hydroxylysine on the biosynthesis of lysine in Saccharomyces. Journal of Bacteriology 108, 715-719.

Somerson, N. L., Demain, A. L. \& Nunheimer, T. D. (I96I). Reversal of lysine inhibition of penicillin production by $\alpha$-aminoadipic acid or adipic acid. Archives of Biochemistry 93, 238-24I.

Trupin, J. S. \& BroQuist, H. P. (1965). Saccharopine, an intermediate of the aminoadipic pathway of lysine biosynthesis. I. Studies in Neurospora crassa. Journal of Biological Chemistry 240, 2524-2530.

TUCCI, A. F. (1969). Feedback inhibition of lysine biosynthesis in yeast. Journal of Bacteriology 99, 624-625.

Yамамото, L. A. \& Segel, I. H. (1966). The inorganic sulfate transport system of Penicillium chrysogenum. Archives of Biochemistry and Biophysics 114, 523-538. 